\title{
Tunneling and subband levels in GaAs quantum well with direct and indirect $A I_{x} \mathrm{Ga}_{1-x} \mathrm{As}$ barriers
}

\author{
Vasu Sankaran and Jasprit Singh \\ Center for High Frequency Microelectronics, Department of Electrical Engineering, and Computer \\ Science, 2234 EECS Building, University of Michigan, Ann Arbor, Michigan 48109-2122
}

(Received 28 June 1991; accepted for publication 22 July 1991)

\begin{abstract}
We present a study of coherent tunneling lifetimes for quasibound electrons confined in a GaAs quantum well by $\mathrm{Al}_{0.3} \mathrm{Ga}_{0.7} \mathrm{As}$ (direct band gap) and AlAs (indirect band gap) barriers, using the tight-binding representation for the electronic states in an eightelement $\left(s p^{3}\right)$ basis, and solving the time-dependent Schrödinger equation using a unitary approximation of the evolution operator. The dependence of the lifetime on barrier thickness is found to fit a WKB-type expression very well. Although simple effective mass theory is not applicable, the barrier thickness coefficient in the WKB exponent is determined by the $\Gamma$-point band extrema even for indirect AlAs barriers with $X$-point conduction-band minimum. The dependence of the subband energies and their in-plane dispersion on the mole fraction $x$ of $\mathrm{Al}$ in the $\mathrm{Al}_{x} \mathrm{Ga}_{1-x} \mathrm{As}$ barrier is also presented, for $x$ in the range $0.2-1$.
\end{abstract}

GaAs and AlAs have an unusual band lineup, since GaAs is a direct band-gap material while AlAs has an indirect band gap. The alloy $\mathrm{Al}_{x} \mathrm{Ga}_{1-x} \mathrm{As}$ has a direct band gap for low Al content $(0 \leqslant x \leqslant 0.35)$, and then has $X$-point conduction-band minima. The tunneling properties of $\mathrm{GaAs} / \mathrm{Al}_{x} \mathrm{Ga}_{1-x}$ As heterostructures have been investigated both for novel device applications as well as for interesting physical insights. Tunneling of electrons from a direct band-gap material such as GaAs through $\mathrm{Al}_{x} \mathrm{Ga}_{1-x} \mathrm{As}$ barriers is described well by WKB-type expressions based on simple single-band effective mass theory, as long as the barrier remains direct. ${ }^{1}$ Several studies in recent years have also focused on tunneling in heterostructures in which the symmetry of the band-edge states differs drastically across the different regions. This is the case, for instance, when a direct band-gap electron ( $\Gamma$ type) escapes through an indirect band-gap barrier ( $X$ or $L$ type). The tunneling transport of electrons from GaAs across a single indirect $\mathrm{Al}_{x} \mathrm{Ga}_{1-x}$ As barrier appears to depend critically on the barrier thickness. For thick barriers, experiments indicate that tunneling is defect assisted, occurring by scattering to the transverse $X$ valleys. ${ }^{2}$ Stationary state calculations also show that for electrons incident from GaAs with energies above the $X$ point but below the $\Gamma$ point of an indirect barrier, the transmission coefficient across sufficiently thick barriers fits an expression of the same functional form as that for transmission over onedimensional rectangular barriers, with the barrier height determined by the $X$ point. But for thinner barriers, tunneling through the $\Gamma$-point states is found to predominate. ${ }^{3,4}$ For incident electron energies below the $\Gamma$ and $X$-band minima, the transmission probability is found to depend exponentially on the energy difference between the carrier energy and the $\Gamma$ minimum in the barrier, even for indirect barriers. ${ }^{5}$

At present no time-domain studies have been reported dealing with electron tunneling through indirect barriers. We have calculated coherent tunneling lifetimes for quasibound electrons escaping from a GaAs quantum well through $\mathrm{Al}_{0.3} \mathrm{Ga}_{0.7} \mathrm{As}$ (direct band gap) and $\mathrm{AlAs}$ (indirect band gap) barriers, such a structure being amenable for experimental work. We address the important question of the role of the $\Gamma$ and $X$ minima in tunneling, and determine the right form of the WKB-type expression that can describe the process for the large $\Gamma$-point band discontinuities that can occur in such structures. We have also examined the eigenvalues, eigenfunctions, and in-plane effective mass of the quantum well ground state for varying alloy composition in the barricr.

We briefly summarize our formalism first, which is discussed in detail elsewhere. ${ }^{6}$ We describe electron states in the conduction band in the tight-binding representation with an eight-element $\left(s p^{3}\right)$ basis. ${ }^{7}$ Since the complete band structure is contained in the tight-binding description, one need not be concerned specifically with the appearance of multiple band extreme or explicit boundary conditions for the components of the electronic wave function. In order to model the escape of electrons from a quantum well through a barrier of finite width, the initial electron wave packet $\psi(\mathbf{r}, 0)$ is constructed as a bound state in the well by extending the barrier. We thus solve the Schrödinger equation:

$$
H \psi(\mathbf{r}, 0)=E \psi(\mathbf{r}, 0)
$$

in the tight-binding representation for the initial state. Subsequently, the time evolution of the state is determined by using a numerically stable and unitary approximation of the evolution operator. ${ }^{8}$ We proceed in infinitesimal time steps $\delta t$, and solve the following equation at each step, once again in the tight-binding representation:

$$
(1+i H \delta t / 2 \hbar) \psi(\mathbf{r}, t+\delta t)=(1-i H \delta t / 2 \hbar) \psi(\mathbf{r}, t) .
$$

Assuming the tunneling escape of carriers to be described by an exponential decay law, tunneling times $\tau$ are obtained from the initial decay rate of probability in the starting well: 

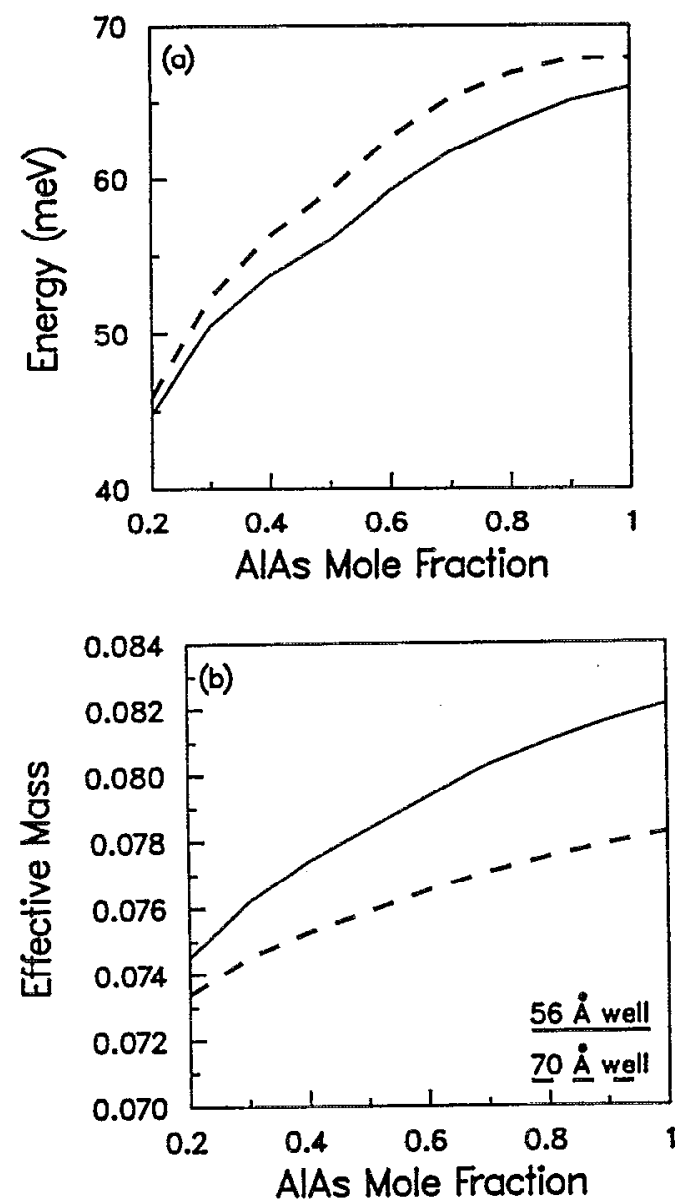

FIG. 1. (a) Variation of the first subband energy level in a $70 \AA ̊ 丿 \mathrm{GaAs}$ quantum well for different alloy compositions of the barrier (solid line). The dotted line is the corresponding result obtained using simple effective mass theory. (b) Dependence of in-plane effective mass on barrier composition for the first electron subband in a GaAs quantum well for two different well sizes.

$$
\tau=\frac{P(0)}{(\Delta P / \Delta t)},
$$

where $P(0)$ is the initial probability in the well and $\Delta P$ the decrease in probability in time $\Delta t$.

The tight-binding wave functions of the first electron subband in the quantum well are primarily $s$ type, but there is a significant $p$-type admixture. This mixture increases as the well size decreases, and can have important effects on the tunneling, transport and optical properties in narrow quantum wells. We note that all the results reported here have been obtained under field-free, flatband conditions. Spin-orbit coupling has been neglected in our computations. Figure 1(a) shows the variation of the ground state energy in a $70 \AA \mathrm{GaAs}$ quantum well for different alloy compositions of the barrier (all energies are measured with respect to the bottom of the conduction band in bulk GaAs). The energy levels are compared with a determinantal solution based on simple effective mass theory, parabolic dispersions, and the $\Gamma-\Gamma$ discontinuity. It is interesting to see that while the agreement between the two is best for direct barriers, the difference between our eightband model and simple effective mass theory is less than
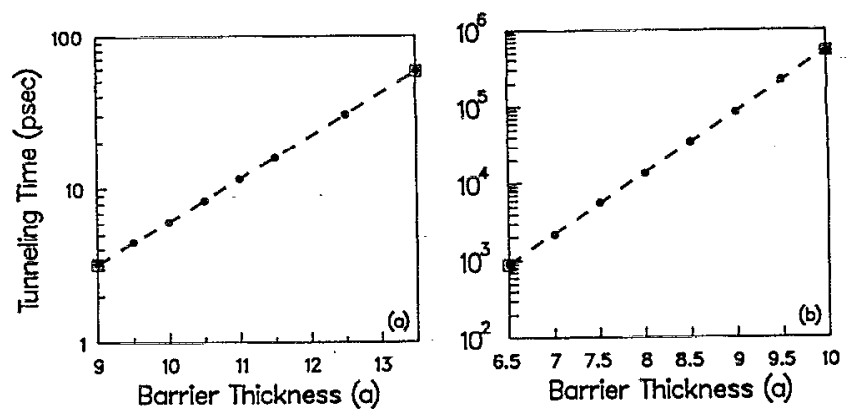

FIG. 2. Tunneling times as a function of barrier thickness for quasibound electrons escaping from a $56 \AA$ GaAs quantum well through barriers of (a) $\mathrm{Al}_{\Theta .3} \mathrm{Ga}_{0.7} \mathrm{As}$ and (b) AlAs. Barrier thickness is in units of the lattice constant. Bullets mark the results of our computations while the dotted line is an exponential fit through them.

$-3 \mathrm{meV}$ over the entire range. The density of states effective mass of carriers in the subband is shown in Fig. 1(b) for two different well widths. The effective masses were obtained by fitting the in-plane dispersion to one that is parabolic in $\mathbf{k}_{\|}$(along $k_{x}$ ), over an energy range of the order of $20 \mathrm{meV}$. The in-plane electron mass is usually taken to be the GaAs band-edge mass $\left(0.067 m_{0}\right)$. We see significant deviation of the mass depending on the barrier material and well size.

Figure 2(a) shows the tunneling times for quasibound electrons escaping from a $56 \AA$ GaAs quantum well through $\mathrm{Al}_{0.3} \mathrm{Ga}_{0.7} \mathrm{As}$ barriers of varying thickness. Figure 2 (b) is the corresponding result with AlAs barriers. The structure considered here is a GaAs quantum well sandwiched between a semi-infinite layer of the barrier material on one side, and a finite barrier followed by a semi-infinite GaAs region on the other. For the range of barrier widths depicted in these figures, the energy $E$ of the quasibound state is only a weak function of the barrier width. This energy is $66.8 \mathrm{meV}$ with $\mathrm{Al}_{0.3} \mathrm{Ga}_{0.7} \mathrm{As}$ barriers, and 91.1 meV with AlAs barriers. We first note that the computed tunneling times fit a single exponential in each figure over the range of barrier widths considered, even at the comparatively long time scales typical of the AlAs barriers. That is, the lifetime $\tau$ for a given type of barrier is found to depend exponentially on the barrier width $L_{b}$ :

$$
\tau=c \exp \left(2 \beta L_{b}\right) \text {. }
$$

That is a standard WKB-type of expression under flatband conditions, in which $2 \beta$ is essentially the decay constant for the probability density, and $\beta$ the decay constant for the exponential decay of the wave function in the barrier. ${ }^{9}$

It is well known that the quantum well eigenstates at a given energy and $\mathbf{k}_{\|}$can be constructed from linear combinations of all bulk eigenstates at the same energy and $\mathbf{k}_{\|}$in each material. The bulk eigenstates at this energy are propagating Bloch states characterized by real wave vector $k_{1}$ in the well material where the subband energy is above the conduction-band minimum, but evanescent states characterized by complex $k_{1}$ in the barrier region where the subband energy falls within the band gap. ${ }^{10,11}$ The complex bands (complex $k_{1}$ ) connect up to real bands (real $k_{1}$ ) at critical points in the bulk bandstructure, as discussed by 
Heine. ${ }^{10}$ The complex bands that are of interest to us for the $\mathrm{Al}_{0.3} \mathrm{Ga}_{0.7} \mathrm{As}$ and $\mathrm{AlAs}$ barriers are the ones that connect to the band extrema at the $\Gamma$ point, and the one emanating from the $X$ point. As we trace the complex contour of $k_{1}$ along any one of these complex bands, the real part of $k_{1}$ does not change significantly from its value at the critical point where it connects to a band extremum. ${ }^{12}$ For the evanescent states, the real part of $k_{1}$ yields an oscillating phase factor, while the imaginary part is responsible for the exponential decay in one direction.

For the $\mathrm{Al}_{0.3} \mathrm{Ga}_{0.7} \mathrm{As}$ barriers, the value of $\beta$ in Eq. (4) that best fits the data was found to be $0.0512 \pi / a$ (where $a$ is the lattice constant). This agrees well with the simple effective mass form

$$
\beta=\sqrt{2 m_{b}\left(V_{b}-E\right)} / \hbar,
$$

where $m_{b}$ is the electron effective mass at the $\Gamma$ point in the barrier $(0.0781)$, and $\left(V_{b}-E\right)$ the energy of the quasibound state with respect to the conduction-band minimum in the barrier. The $\Gamma-\Gamma$ barrier height $\left(V_{b}\right)$ was set to 250 $\mathrm{meV}$ in our computations. This agreement reaffirms the fact that for direct barriers a more complete description of the electron state yields a tunneling time that compares well with WKB estimates based on simple effective mass theory.

For the AlAs barriers $\beta$ was found to be $0.1462 \pi / a$, which does not fit a simple effective mass form as in the above expression. The electron effective mass and barrier height at the $\Gamma$ point were 0.1548 and $1.15 \mathrm{eV}$, respectively. The corresponding values at the $X$ point were 1.3514 and $190 \mathrm{meV}$, respectively. On the other hand, we find that the bulk evanescent state in AlAs obtained from our tight-binding Hamiltonian with complex $k_{\perp}=i 0.1462 \pi / a=i \beta$ is at the same energy below the $\Gamma$ point conduction-band minimum as the quasibound state $(1.15-0.0911=1.059 \mathrm{eV})$. As noted earlier, the imaginary part of $k_{1}$ is the decay constant for the exponential decay of the bulk tight-binding state in one direction, and this is the factor that we would expect in the WKB expression. But the simple form in Eq. (5) does not yield the correct imaginary wave vector of the band-gap state because the quasibound state is about $1 \mathrm{eV}$ below the band edge, and simple effective mass theory is not expected to hold in this range. We now show that a Kane-type, two band k.p model of the band structure near the $\Gamma$ point is all that is required to describe the barrier states. ${ }^{13}$

In the absence of spin-orbit coupling the valence-band structure along (100) consists of a doubly degenerate heavy-mass band, and a light-mass band, with triple degen- eracy at the zone center. The evanescent state with $k_{1}=i 0.1462 \pi / a$ lies on the complex $k_{1}$ contour that connects the top of the light-mass band to the bottom of the conduction band at the $\Gamma$ point. The dispersion of these two bands near the $\Gamma$ point can be described in terms of a $2 \times 2 \mathrm{k} \cdot \mathrm{p}$ Hamiltonian obtained from Kane's four-band Hamiltonian ${ }^{13}$ by setting $\mathbf{k}_{\|}=0$ :

$H\left(k_{z}\right)=\left[\begin{array}{cc}E_{c}+\frac{\hbar^{2} k_{z}^{2}}{2 m_{0}}+A^{\prime} k_{z}^{2} & i P k_{z} \\ -i P k_{z} & E_{v}+\frac{\hbar^{2} k_{z}^{2}}{2 m_{0}}+L^{\prime} k_{z}^{2}\end{array}\right]$,

with the same notation as in Ref. 13. Our tight-binding parameters of AlAs yield a band gap of $3.3316 \mathrm{eV}$ at the $\Gamma$ point. We fit the parameters $A^{\prime}, L^{\prime}$, and $P$ above to yield the electron and light-hole effective masses of 0.1548 and 0.1486 , respectively. Using this Hamiltonian to solve for an eigenstate at an energy of $1.059 \mathrm{eV}$ below $E_{c}$ yields a wave vector $k_{z}=i 0.1492 \pi / a$, which compares very well with the decay constant obtained from the WKB expression.

In summary, we have calculated coherent tunneling lifetimes for quasibound electrons escaping from a $\mathrm{GaAs}$ quantum well through $\mathrm{Al}_{0.3} \mathrm{Ga}_{0.7} \mathrm{As}$ (direct band gap) and AlAs (indirect band gap) barriers. The escape times through the direct barrier fit a WKB expression based on simple effective mass theory. The escape times through the indirect barrier aiso fit a WKB expression with an exponential dependence on the barrier width. While the decay factor in the exponential does not fit a simple effective mass form, it can be derived from a two-band $\mathbf{k} \cdot \mathbf{p}$ Hamiltonian at the $\Gamma$ point, with no explicit contribution from the $X$ point states.

This work was supported by the U.S. Army Research Office (grant No. DAAL03-87-K-0007).

'T. B. Norris, X. J. Song, W. J. Schaff, L. F. Eastman, G. Wicks, and G. A. Mourou, Appl. Phys. Lett. 54, 60 (1989).

${ }^{2}$ P. M. Solomon, S. L. Wright, and C. Lanza, Superlatt. Microstruct. 2, 521 (1986).

${ }^{3}$ G. C. Osbourn, J. Vac. Sci. Technol. 19, 592 (1981).

${ }^{4}$ C. Mailhiot, T. C. McGill, and J. N. Schulman, J. Vac. Sci. Technol. B 1, 439 (1983).

${ }^{5}$ G. C. Osbourn, J. Vac. Sci. Technol. 17, 1104 (1980).

${ }^{6} \mathrm{~V}$. Sankaran and J. Singh, Phys. Rev. B 44, 3175 (1991).

${ }^{7}$ J. C. Slater and G. F. Koster, Phys. Rev. 94, 1498 (1954).

${ }^{8}$ A. Goldberg, H. M. Schey, and J. L. Schwartz, Am. J. Phys. 35, 177 (1967).

${ }^{9}$ L. I. Schiff, Quantum Mechanics (McGraw-Hill, New York, 1949).

${ }^{10}$ V. Heine, Proc. Phys. Soc. Iondon 81, 300 (1963).

${ }^{11}$ G. C. Osbourn and D. L. Smith, Phys. Rev. B 19, 2124 (1979).

${ }^{12}$ Y-C. Chang, Phys. Rev. B 25, 605 (1982).

${ }^{13}$ E. O. Kane, in Semiconductors and Semimetals, edited by R. K. Willardson and A. C. Beer (Academic, New York, 1966), Vol. 1, p. 75. 